\title{
Os Desafios dos Hospitais Perante a COVID-19 e a Gripe Sazonal Durante o Outono-Inverno de 2020/2021
}

\author{
COVID-19 and Seasonal Flu During the Autumn-Winter \\ of 2020/2021 and the Challenges Lying Ahead for \\ Hospitals
}

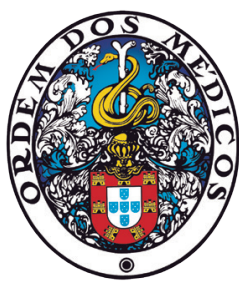

Luís CAMPOS $\square^{1,2}$, Kamal MANSINHO ${ }^{1,2}$, Paulo TELLES DE FREITAS ${ }^{3}$, Victor RAMOS ${ }^{4}$, Constantino SAKELLARIDES ${ }^{4}$ Acta Med Port 2020 Nov;33(11):716-719 - https://doi.org/10.20344/amp.14818

RESUMO

A possibilidade da coexistência de uma segunda vaga da pandemia de COVID-19, com uma epidemia simultânea de gripe e de co-circulação de outros vírus respiratórios sazonais cria o cenário para uma tempestade perfeita. A preparação do Outono-Inverno de 2020/2021 é complexa, exige orientações centralizadas mas soluções locais e regionais, com forte liderança e elevado nível de coordenação. É essencial actuar a montante dos hospitais para diminuir o afluxo às urgências, minimizando o risco de transmissão que aí ocorre e a sobrecarga das equipas, a jusante para garantir capacidade de internamento e no próprio hospital para optimizar os recursos e a organização. A falência deste plano originará uma pressão insuportável nos cuidados hospitalares. Os autores enunciam os desafios que os hospitais enfrentam e as principais medidas que deverão fazer parte desse plano para preparar o Outono-Inverno de 2020/2021, em Portugal.

Palavras-chave: COVID-19; Estações; Influenza Humana; Serviço Hospitalar de Emergência

\section{ABSTRACT}

The possibility of a second wave of the COVID-19 pandemic coexisting with a simultaneous epidemic of influenza and the co-circulation of other seasonal respiratory viruses sets the stage for a perfect storm. Preparing for the Autumn-Winter of 2020/2021 is complex, requiring centralized guidance but local and regional solutions, with strong leadership and a high level of coordination. It is essential to act upstream of hospitals in order to reduce demand on emergency departments, minimizing the risk of transmission that occurs there and the team overload, as well as downstream to ensure capacity for hospitalization and in the hospital itself to optimize resources and organization. The failure of this plan will create unbearable pressure on hospital care. The authors describe the challenges lying ahead for hospitals and the most important measures that should be included in this plan to prepare the Autumn-Winter of 2020/2021 in Portugal. Keywords: COVID-19; Emergency Service, Hospital; Influenza, Human; Seasons

\section{INTRODUÇÃO}

Pela primeira vez na história moderna, o hemisfério norte enfrenta a possibilidade da coexistência de uma segunda vaga da pandemia de COVID-19, com uma epidemia simultânea de gripe e de co-circulação de outros vírus respiratórios sazonais. Qualquer destes vírus pode provocar doença grave e morte, especialmente em pessoas mais vulneráveis. ${ }^{1}$ Assim, o próximo Inverno representa um desafio adicional para os cuidados de saúde, e em particular para os hospitais, onde confluem graves disfunções do sistema de saúde: recursos insuficientes, fragmentação (não integração e continuidade) dos cuidados, modelos de decisão centralizados e ausência de uma política para as profissões que atraia e motive os profissionais do Serviço Nacional de Saúde (SNS). Durante o Verão, muitos hospitais já se debatiam com insuficiência de recursos humanos, existindo camas fechadas e serviços superlotados.

Apesar de persistirem consideráveis incertezas em relação ao comportamento sazonal do SARS-CoV-2 e da relação temporal, dimensão e gravidade da coexistência desta pandemia com a gripe sazonal, pode acontecer que as medidas preventivas tomadas diminuam o impacto da gripe sazonal, tal como tem acontecido em alguns países do hemisfério Sul. Mas se isso não acontecer, enfrentamos a possibilidade de uma tempestade perfeita num sector onde cada Inverno gera uma crise. Alguns modelos estimam, entre setembro de 2020 e junho de 2021 , o dobro das mortes no hospital do que as que ocorreram na Primavera de $2020 .^{2}$ Alguns países têm publicado relatórios técnicos solicitados a grupos ou comissões de peritos qualificados para o efeito, relativos ao próximo Outono e Inverno. ${ }^{3-5}$

No nosso país, o Ministério da Saúde divulgou, a 21 de setembro, um Plano da Saúde para o Outono-Inverno de 2020-2021 que, genericamente, recomenda medidas consensuais, sendo algumas coincidentes com as apresentadas neste artigo. No entanto, este plano já é tardio e ainda tem que ser apreciado pelo Conselho Económico e Social e pelo Conselho Nacional de Saúde. ${ }^{6}$ Além disso, é muito intencional e pouco operacional, verificando-se a ausência de um enquadramento estratégico, priorização das medidas, quantificação, um cronograma, definição

1. Hospital São Francisco Xavier. Centro Hospitalar Lisboa Ocidental. Lisboa. Portugal.

2. NOVA Medical School/Faculdade de Ciências Médicas. Lisboa. Portugal.

3. Hospital Fernando da Fonseca. Amadora. Portugal.

4. Escola Nacional de Saúde Pública. Lisboa. Portugal.

$\triangle$ Autor correspondente: Luís Campos. Imcampos@chlo.min-saude.pt

Recebido: 28 de agosto de 2020 - Aceite: 28 de agosto de 2020 | Copyright @ Ordem dos Médicos 2020 
de responsabilidades, financiamento associado, gestão dos recursos humanos e procedimentos em caso de sobrelotação. Finalmente, não presta atenção às necessidades dos profissionais de saúde, muito acentuadas por esta crise, e as abordagens referentes à participação dos cidadãos são muito insuficientes.

Em Portugal, que é o país europeu onde as pessoas recorrem mais às urgências hospitalares, ${ }^{7}$ estas poderão colapsar se não atuarmos a montante. O internamento irá transbordar se não interviermos a jusante. Entraremos numa nova fase de cancelamento da atividade programada com consequências catastróficas para a saúde das populações. ${ }^{8}$

O hospital tem que se reorganizar internamente para responder a estes desafios. Este é um empreendimento que tem que ser preparado com antecedência, tirando proveito da experiência recente, tanto nacional como internacional. O seu sucesso exige um aumento da capacidade de interação com o sistema de saúde e a sua preparação deve fazer parte de uma estratégia de saúde integrada e faseada, de acordo com a previsível evolução da pandemia. Este plano deve ser construído de forma participada, exige um acordo mínimo com as profissões, e um compromisso multissectorial entre os principais parceiros envolvidos.

Estes são tempos excecionais, que requerem respostas excecionais: os modelos conhecidos de operar foram interrompidos e o novo normal ainda não emergiu. ${ }^{9}$ Esta é uma oportunidade para resolução de problemas há muito identificados. Precisamos de voltar ao futuro e não ao passado. De seguida apontamos as principais medidas que consideramos que deverão fazer parte desse plano para preparar o Inverno de 2020/2021, em Portugal.

\section{Antes do hospital}

Será oportuna uma estratégia de comunicação apropriada focada nos desafios do Outono/Inverno. É necessário minimizar a transmissão do SARS-Cov-2 e do vírus influenza com um foco particular na importância dos comportamentos individuais, implementar medidas de prevenção e mitigação da COVID-19, executar políticas pró-ativas de proteção dos grupos mais vulneráveis, manter um esforço de testagem intenso, otimizar o controlo dos surtos, reforçar a capacidade das unidades de Saúde Pública, prevenir a infeção nosocomial, unificar registos, melhorar a eficiência dos sistemas de informação (SINAVE e Trace COVID-19), partilhar os dados e reforçar a vacinação para a gripe, com atenção especial aos profissionais de saúde e do sector social, procurando reduzir o afluxo às urgências destes doentes.

O SNS necessita de um dispositivo de regulação do acesso, sendo o acesso aos serviços de urgência uma componente particularmente crítica. Este dispositivo deveria obedecer a um conjunto de princípios gerais, mas as soluções deveriam ser locais e regionais de acordo com experiências anteriores e contextos específicos.

Será necessário incentivar junto da população uma utilização mais racional das urgências, promover o recurso à linha Saúde 24, aumentar a capacidade resolutiva dos cuidados de saúde primários e a sua disponibilidade para atender as situações agudas não urgentes, alargar horários de atendimento, reservar vagas nas consultas hospitalares para atendimentos não programados, promover uma meIhor utilização dos hospitais de dia e lançar programas de gestão de caso para os utilizadores frequentes das urgências.

De forma a descongestionar os serviços de urgência será essencial permitir o atendimento aos doentes com sintomas gripais ou infeções respiratórias em estruturas dedicadas fora do espaço das urgências. Estas unidades, que já foram ensaiadas durante a pandemia da gripe $A$ em 2009, deveriam ser asseguradas segundo uma formulação organizacional a acordar localmente entre os hospitais e os cuidados de saúde primários. Estas unidades são essenciais e estão preconizadas no Plano do Ministério com a designação de Áreas Dedicadas para Doentes Respiratórios. A implementação de testes rápidos para os vários vírus respiratórios seria importante para acelerar o fluxo dos doentes.

\section{No hospital}

Na sua organização interna, o hospital terá que se reestruturar para manter níveis de prontidão, sazonais ou não, de acordo com as dinâmicas epidémicas ou pandémicas e, simultaneamente, garantir a assistência à população geral, tendencialmente envelhecida, com múltiplas comorbilidades crónicas.

Devem existir planos de contingência elaborados com o envolvimento dos profissionais 'no terreno', com respostas planificadas para os vários cenários e com atribuição clara de funções (consultivas e normativas) e responsabilidades. Estes planos devem ser do conhecimento de todos os profissionais, para que todos saibam o quê, quando, porquê e a quem.

O atendimento dos doentes sem situações urgentes, (verdes e azuis da triagem de Manchester) deve ser separado do dos doentes com situações urgentes e emergentes. Estes doentes devem ser reencaminhados para os centros de saúde, os quais devem garantir disponibilidade para os observar num tempo adequado.

Durante o inverno, os hospitais devem adotar uma lógica explícita sobre o que podem fazer e sobre aquilo que não farão. Não podem adiar tudo. Uma das lições mais relevantes dos surtos de SARS em Toronto, de cólera no Haiti, de MERS na Coreia do Sul e de Zika nas Américas e reforçada pela atual pandemia de COVID-19, destaca que é incongruente impedir os óbitos causados por epidemias em curso, quando um número elevado de outros doentes morre porque não obteve, atempadamente, cuidados médi$\cos ^{10}$

A nível funcional, haverá que evitar a transmissão nosocomial, através de circuitos e áreas de utilização corretamente sinalizadas ("não deixar que a infeção tome conta do hospital"), melhorar a qualidade do trabalho de equipa, através da partilha contínua da informação e decisão, 
tos e procedimentos.

combater a fadiga e o burnout, inovando procedimentos e alternando funções e atualizar continuamente conhecimen-

A estrutura dos hospitais em silos estanques dedicados a órgãos ou sistemas é inadequada. Essa inadequação é ainda mais manifesta perante uma pandemia como esta. Promover uma organização mais matricial, baseada em departamentos com uma gestão comum de camas é mais flexível e adequada para responder à variabilidade da necessidade de camas de internamento.

As camas hospitalares devem ter tipologias diferenciadas em função da gravidade dos doentes. As camas COVID e não-COVID devem ser identificadas, com circuitos separados, estabelecendo as capacidades máximas de internamento para cada instituição, nas suas diferentes tipologias.

Os profissionais que estão na linha da frente devem poder descansar para estarem preparados e devem ser justamente compensados. Muitos estão exaustos e desmotivados. É preciso colmatar os défices que persistem em alguns sectores e captar para o SNS os recém- especialistas das especialidades que têm estado na linha da frente ou no seu apoio. A Medicina Interna, a Infeciologia, a Pneumolo- gia, a Anestesiologia e a Medicina Intensiva em particular, são as especialidades que têm garantido a assistência à maior parte dos doentes COVID-19 internados nas enfermarias e em cuidados intensivos e têm assegurado o seu atendimento nas urgências externas. Em situações limite, todas as especialidades podem ser recrutadas assim como os alunos de Medicina do sexto ano. É imprevisível também o impacto da ausência ao trabalho dos profissionais de saúde por motivo de doença. Vai ser muito importante o papel dos serviços de Saúde Ocupacional, que devem ser reforçados. A cobertura assistencial deve ser homogénea, ao longo da semana, com reengenharia de horários para garantir os dias de descanso.

Também devem ser ampliados, na medida do possível, os programas de hospitalização domiciliária, que evitam a admissão em enfermarias hospitalares de muitos doentes que podem ser tratados em casa por equipas hospitalares.

Os orçamentos-programa contratualizados com os hospitais estão ultrapassados pela paragem das atividades programadas. O não cumprimento dos objetivos iniciais não pode comprometer o financiamento dos hospitais, que tiveram que incorrer em despesas extraordinárias.

Tabela 1 - Medidas a tomar nos hospitais, a montante e a juzante para enfrentar o Outono-Inverno de 2020/2021

\begin{tabular}{|c|c|c|}
\hline Antes do hospital & No hospital & Depois do hospital \\
\hline 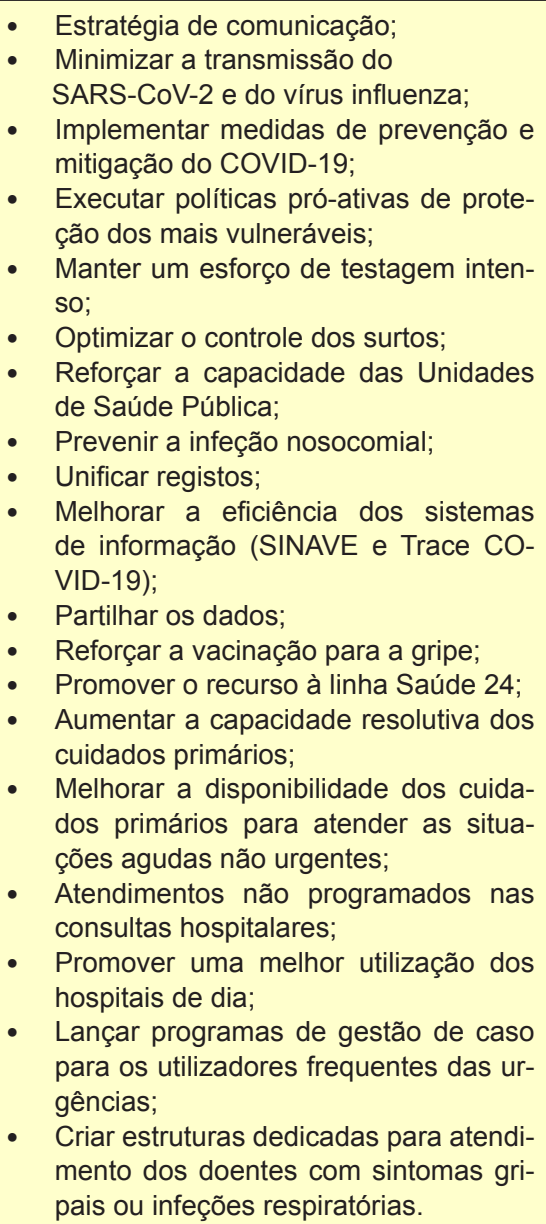 & $\begin{array}{l}\text { - Incrementar a flexibilidade e a escala- } \\
\text { bilidade das estruturas; } \\
\text { - Assegurar a existência de planos de } \\
\text { contingência; } \\
\text { - Manter uma reserva estratégica de } \\
\text { equipamentos; } \\
\text { - Garantir a manutenção da assistência } \\
\text { aos doentes não COVID; } \\
\text { - Atender fora das urgências os doentes } \\
\text { sem situações urgentes ou emergen- } \\
\text { tes; } \\
\text { - Definir circuitos e áreas para doentes } \\
\text { COVID e não COVID; } \\
\text { - Promover medidas que previnam a fa- } \\
\text { diga e o burnout dos profissionais; } \\
\text { - Estabelecer as capacidades máximas } \\
\text { de internamento nas diferentes tipolo- } \\
\text { gias de camas; } \\
\text { - Colmatar os défices de recursos huma- } \\
\text { nos; } \\
\text { - Prever reforço extraordinário de recur- } \\
\text { - } \text { sos humanos; } \\
\text { Promover uma gestão comum de ca- } \\
\text { mas; } \\
\text { - } \text { Implementar uma organização depar- } \\
\text { tamental; } \\
\text { ção domiar os programas de hospitaliza- } \\
\text { Rever os orçamentos programa. }\end{array}$ & $\begin{array}{l}\text { - Criar alternativas para retirar dos hos- } \\
\text { pitais os doentes sociais ou à espera } \\
\text { de vaga na RNCCI; } \\
\text { - Criar estruturas que recebam os doen- } \\
\text { tes COVID que permanecem interna- } \\
\text { dos apenas por não poderem fazer a } \\
\text { quarentena em casa; } \\
\text { - Fazer uma gestão regional da capaci- } \\
\text { dade de internamento; } \\
\text { - Criar estruturas permanentes de coor- } \\
\text { denação entre hospitais, cuidados pri- } \\
\text { mários, continuados e paliativos, ação } \\
\text { social, autarquias e recursos na comu- } \\
\text { nidade. }\end{array}$ \\
\hline
\end{tabular}




\section{Depois do hospital}

Para aumentar a capacidade de internamento é necessário criar alternativas para acolher os doentes com alta clínica, que permanecem nas enfermarias (em fevereiro de 2020 eram 1551), seja por motivos sociais ou à espera de vaga na Rede Nacional de Cuidados Continuados Integrados (RNCCl). O mesmo se aplica aos doentes com COVID-19, que se mantêm internados por razões sociais ou porque não têm condições em casa para cumprir a quarentena ( $18 \%$ dos 810 doentes internados em maio). Estas soluções devem envolver as autarquias e o sector social. ${ }^{11}$

Os serviços que recebem os doentes COVID-19 são os que têm também de admitir os doentes com complicações da gripe ou com outras infeções respiratórias. Num cenário pior é expectável que os hospitais esgotem a sua capacidade de internamento. É necessário fazer uma gestão regional da capacidade de internamento e ter disponíveis alternativas externas à rede do SNS, com camas, equipamentos e recursos humanos suficientes (hospitais militares, do sector social ou mesmo privados - ver Tabela 1).

\section{Uma visão de conjunto}

Os hospitais não podem ser abandonados à sua sorte. Tudo o que correr mal ou não for feito na preparação do próximo Outono-Inverno vai recair sobre as urgências e internamento hospitalares, o último recurso dos doentes. São necessárias orientações nacionais que abranjam o conjunto do sistema de saúde, coordenações regionais e sub-regionais, particularmente nas áreas metropolitanas de
Lisboa e Porto, e soluções locais validadas e monitorizadas. Isso incluirá hospitais, cuidados de saúde primários, continuados e paliativos, ação social e autárquica e requer recursos na comunidade, com lideranças competentes, capacidade de decisão e meios de resposta. Os vários níveis de cuidados devem ser geridos de forma coordenada, com processos integrados e melhor comunicação entre eles.

Os hospitais continuam a ser polos de ensino pré-graduado, escolas de formação pós-graduada e centros de investigação clínica, sendo, por excelência, uma interface privilegiada com a academia, com a investigação e o desenvolvimento do País, pelo que devem ter condições de manter estas missões.

A resposta hospitalar à pandemia, deve integrar-se numa estratégia de saúde para o país (2020-2021), que articule todas as respostas do sistema de saúde, compaginando-as com o desenvolvimento económico e social do país. Esta estratégia é de natureza contingencial, contemplando o faseamento que a evolução da pandemia impõe. ${ }^{12}$ O que se fará no Outono tem a ver com como chegámos ao fim do Verão. As respostas na próxima Primavera dependem de como chegamos ao fim do Inverno. A comunicação é um componente essencial dessa estratégia. Não basta informar, é necessário analisar o seu impacto real e aprender com isso.

Para desafios complexos é necessário pensar e agir de forma sistémica, integrada e nos tempos próprios, para que todos cumpram o papel que thes cabe.

OECD Publishing; 2015

8. Nogueira PJ, Nobre MA, Nicola PJ, Furtado C, Carneiro AV. Excess mortality estimation during the COVID-19 pandemic: preliminary data from Portugal. Acta Med Port. 2020;33:376-83.

9. Imperial College. Leading and managing during COVID-19 crisis. [consultado 2020 jun 10]. Disponível em: https://www.imperial. ac.uk/staff-development/learning-and-development-centre/courses/ leadership/covid/.

10. Reperant LA, Osterhaus AD. AIDS, avian flu, SARS, MERS, Ebola, Zika... what next? Vaccine. 2017;35:4470-4.

11. Barómetro de internamentos sociais \& COVID-19, 2020. [consultado 2020 ago 20]. Disponível em: https://apah.pt/noticia/barometro-deinternamentos-sociais-revela-1500-pessoas-nos-hospitais-nestasituacao-representando-184me/.

12. Sakellarides $C$, Araujo F. Estratégia de Saúde Pública para a pandemia COVID-19 em Portugal: contribuições da experiência internacional. Acta Med Port. 2020;33:456-8. 